\title{
Review of: "Visualizing sequential compound fusion and kiss-and-run in live excitable cells"
}

\author{
Lei $\mathrm{Xue}^{1}$
}

1 Fudan University

Potential competing interests: The author(s) declared that no potential competing interests exist.

In this study, Ge et al. established the concept of sequential compound fusion and compound kiss-and-run by directly visualizing their membrane, fusion pore, and content-release dynamics in live cells for the first time. Furthermore, they found that sequential compound fusion and compound kiss-and-run are supposed to constitute novel mechanisms contributing to the generation of desynchronized multi-vesicular release at single release sites, asynchronous release, vesicle docking/priming, and vesicle endocytosis. I just have a few comments on the current manuscript.

1. The authors visualized sequential compound fusion and compound kiss-and-run in primary cultured bovine adrenal chromaffin cells, which belong to neuroendocrine cells. However, whether the novel vesicle fusion phenomenon also exists in neurons and its potential physiological significance is unclear. Although currently, it is tough to verify these points in neurons, the authors should at least discuss them.

2. It is generally accepted that calcium influx regulates exocytosis in endocrine cells and neurons (Weiss and Zamponi, 2013; Gabel et al., 2020). I wonder whether the novel fusion modes sequential compound fusion and compound kiss-and-run are also calcium-dependent. In addition, it will be interesting to examine whether calmodulin and/or calcineurin, which locate the downstream of calcium, can also modulate these novel fusion modes.

3. SNARE proteins are suggested to be required during vesicle fusion with the presynaptic membrane (Chen, Scales, and Scheller, 2001; Sharma and Lindau, 2018). The discussion section in this study mentioned that a possible mechanism of sequential compound fusion is due to the diffusion of TSNARE from the plasma membrane to the vesicle. However, this study showed that some vesicles may undergo sequential compound fusion within 0.2 seconds. I doubt that whether the T-SNARE can diffuse from the plasma membrane to the vesicle in such a short time.

4. L.G.'s group has reported the compound fusion at the calyx-type synapse upon intense stimulation (He et al., 2009). Therefore, I wonder whether the sequential compound fusion and compound kiss-and-run are also activity-dependent. A further investigation would help clarify the physiological significance of these novel fusion modes.

5. L.G.'s group has reported the fusion pore behaviors in live cells. The diverse pore dynamics crucially 
determine the efficiency of vesicle release and retrieval (Shin et al., 2018). Similarly, it would be informative to know the dynamic fusion pore behaviors during sequential compound fusion and compound kiss-and-run, respectively. In addition, it would be interesting to know whether the dynamic fusion pore behaviors of the first vesicle that fuses with the presynaptic membrane can be modified by the sequential compound fusion.

\section{References}

Chen, Y. A., S. J. Scales, and R. H. Scheller. 2001. 'Sequential SNARE assembly underlies priming and triggering of exocytosis', Neuron, 30: 161-70.

Gabel, M., C. Royer, T. Thahouly, V. Calco, S. Gasman, M. F. Bader, N. Vitale, and S. Chasserot-Golaz. 2020. 'Annexin A2 Egress during Calcium-Regulated Exocytosis in Neuroendocrine Cells', Cells, 9.

He, L., L. Xue, J. Xu, B. D. McNeil, L. Bai, E. Melicoff, R. Adachi, and L. G. Wu. 2009. 'Compound vesicle fusion increases quantal size and potentiates synaptic transmission', Nature, 459: 93-7.

Sharma, S., and M. Lindau. 2018. 'Molecular mechanism of fusion pore formation driven by the neuronal SNARE complex', Proc Natl Acad Sci U S A, 115: 12751-56.

Shin, W., L. Ge, G. Arpino, S. A. Villarreal, E. Hamid, H. Liu, W. D. Zhao, P. J. Wen, H. C. Chiang, and L. G. Wu. 2018. 'Visualization of Membrane Pore in Live Cells Reveals a Dynamic-Pore Theory Governing Fusion and Endocytosis', Cell, 173: 934-45.e12.

Weiss, N., and G. W. Zamponi. 2013. 'Control of low-threshold exocytosis by T-type calcium channels', Biochim Biophys Acta, 1828: 1579-86. 